\title{
Multiple White Flat Lesions of the Corpus: Subtype of Hyperplastic Polyps vs. Intestinal Metaplasia
}

\author{
Su Jin Kim and Cheol Woong Choi \\ Department of Internal Medicine, Pusan National University School of Medicine and Research Institute for Convergence of Biomedical \\ Science and Technology, Pusan National University Yangsan Hospital, Yangsan, Korea
}

See "Magnified Endoscopic Findings of Multiple White Flat Lesions: A New Subtype of Gastric Hyperplastic Polyps in the Stomach" by Rino Hasegawa, Kenshi Yao, Shoutomi Ihara, et al., on page 558-562.

Magnifying endoscopy with narrow-band imaging (MENBI) provides information on the microsurface structure and microvascular architecture of the mucosa. ${ }^{1}$ The vessel plus surface classification that uses this information improves the diagnostic accuracy of endoscopy for early gastric cancer. ${ }^{2}$ ME-NBI is also applicable in the diagnosis of gastric mucosa-associated lymphoid tissue lymphoma, chronic gastritis, and intestinal metaplasia (IM). ${ }^{3-5}$ The superior diagnostic accuracy of ME-NBI compared with that of white light endoscopy of gastric IM can aid in determining the surveillance interval based on gastric cancer risk stratification, such as the operative link of gastric IM assessment staging system. ${ }^{6}$ Therefore, the differential diagnosis with ME-NBI of whitish flat elevated lesions between IM and other benign lesions, including fundic gland and hyperplastic polyps, is important.

In a current issue of Clinical Endoscopy, Hasegawa et al. reported the ME-NBI and clinicopathologic findings of multiple whitish flat elevated lesions (MWFLs) extending from

Received: September 19, 2018 Revised: October 5, 2018

Accepted: October 5, 2018

Correspondence: Cheol Woong Choi

Department of Internal Medicine, Pusan National University School of Medicine and Research Institute for Convergence of Biomedical Science and Technology, Pusan National University Yangsan Hospital, 20 Geumo-ro, Mulgeum-eup, Yangsan 50612, Korea

Tel: +82-55-360-1535, Fax: +82-55-360-1536, E-mail: drluckyace@gmail.com ORCID: https://orcid.org/0000-0001-8867-3039

cc This is an Open Access article distributed under the terms of the Creative Commons Attribution Non-Commercial License (http://creativecommons.org/ licenses/by-nc/3.0) which permits unrestricted non-commercial use, distribution, and reproduction in any medium, provided the original work is properly cited. the gastric body to the fundus. ${ }^{7}$ A previous study evaluating the endoscopic classification, using ME-NBI, of gastric precancerous and cancerous lesions proposed that lesions with tubular-villous mucosal and regular vascular patterns can be classified as IM. ${ }^{8,9}$ The authors reported that the false-positive rate was about $10 \%$ (10 of 100 IM cases diagnosed using ME$\mathrm{NBI}$ ) and that foveolar hyperplasia presenting with this MENBI pattern may be the cause of false-positive results. ${ }^{10}$ In conference abstracts from Japanese endoscopists, MWFLs were reported as independent entities, not IM. ${ }^{11,12}$ The common histologic findings of these lesions included straight enlarged foveolar epithelium with atrophy of the fundic gland without cystic dilatation. Hasegawa et al. also reported observing foveolar hyperplasia in $100 \%$ of 4 patients, and parietal cell protrusion and oxyntic gland dilatation in $75 \%$ of the patients. ${ }^{7}$ Fundic gland and hyperplastic polyps induced by proton pump inhibitor use have different histologic features from those of MWFLs. Fundic gland polyps are characterized by cystic dilatation of pits, which are located in deep mucous neck cells. ${ }^{13}$ Hyperplastic polyps are associated with hypergastrinemia and Helicobacter pylori infection, and have histologic characteristics of elongation, twisting, branching, and cystic dilatation of the foveolae. ${ }^{14}$

ME-NBI findings showed uniformly long and slightly wide marginal crypt epithelium and a faint dark brown color in the intervening part. There are 3 points in the differential diagnosis of IM. First, MWFLs present as whitish elevated patches in the upper corpus, whereas whitish elevated lesions on the antrum are usually IM. Second, this lesion can be observed 
without atrophic background because $H$. pylori infection, which causes atrophic gastritis, is present in $7.7 \%-31.7 \%$ of the patients. ${ }^{7}$ IM, the replacement of the gastric mucosa by intestinal epithelium, usually develops after atrophic change. Third, MWFLs, based on ME-NBI findings, have papillary or ridged marginal crypt epithelium without the light-blue crest sign, which is an endoscopic diagnostic indicator of $\mathrm{IM}^{5}$ However, the most accurate diagnostic method is histologic assessment of biopsy specimens. The above-mentioned 3 points for differential diagnosis are based on the features of IM. Clinicians must remember that although these findings may increase the accuracy of endoscopic diagnosis, they may also be false negatives. Of course, biopsy has limitations, such as sampling errors, additional expense, and being time consuming. Further studies are needed to evaluate the clinical and pathologic characteristics of MWFLs to overcome these limitations.

In conclusion, MWFLs of the corpus can be considered as a subtype of hyperplastic polyps. Especially, a misdiagnosis of this lesion as IM causes patients to be classified as having a high risk for gastric cancer. Therefore, identification of the differences between MWFLs and IM is important.

\section{Conflicts of Interest}

The authors have no financial conflicts of interest.

\section{Author Contributions}

Conceptualization: Su Jin Kim

Supervision: Cheol Woong Choi

Writing-original draft: SJK

Writing-review \& editing: CWC

\section{REFERENCES}

1. Yao K. Clinical application of magnifying endoscopy with narrow-band imaging in the stomach. Clin Endosc 2015;48:481-490.

2. Yao K, Anagnostopoulos GK, Ragunath K. Magnifying endoscopy for diagnosing and delineating early gastric cancer. Endoscopy 2009;41:462467.

3. Ono S, Kato M, Ono Y, et al. Characteristics of magnified endoscopic images of gastric extranodal marginal zone B-cell lymphoma of the mucosa-associated lymphoid tissue, including changes after treatment. Gastrointest Endosc 2008;68:624-631.

4. Anagnostopoulos GK, Yao K, Kaye P, et al. High-resolution magnification endoscopy can reliably identify normal gastric mucosa, Helicobacter pylori-associated gastritis, and gastric atrophy. Endoscopy 2007;39:202-207.

5. Uedo $\mathrm{N}$, Ishihara $\mathrm{R}$, Iishi $\mathrm{H}$, et al. A new method of diagnosing gastric intestinal metaplasia: narrow-band imaging with magnifying endoscopy. Endoscopy 2006;38:819-824.

6. Saka A, Yagi K, Nimura S. OLGA- and OLGIM-based staging of gastritis using narrow-band imaging magnifying endoscopy. Dig Endosc 2015;27:734-741.

7. Hasegawa R, Yao K, Ihara S, et al. Magnified endoscopic findings of multiple white flat lesions: a new subtype of gastric hyperplastic polyps in the stomach. Clin Endosc 2018;51:558-562.

8. Pimentel-Nunes P, Dinis-Ribeiro M, Soares JB, et al. A multicenter validation of an endoscopic classification with narrow band imaging for gastric precancerous and cancerous lesions. Endoscopy 2012;44:236-246.

9. Pimentel-Nunes P, Libânio D, Lage J, et al. A multicenter prospective study of the real-time use of narrow-band imaging in the diagnosis of premalignant gastric conditions and lesions. Endoscopy 2016;48:723730 .

10. Pimentel-Nunes P, Dobru D, Libânio D, Dinis-Ribeiro M. White flat lesions in the gastric corpus may be intestinal metaplasia. Endoscopy 2017;49:617-618.

11. Kamada T, Kawaguchi M, Maruyama Y, et al. New gastric lesion in the cardia induced by proton pump inhibitor treatment. Gastroenterology 2011;140(5 Suppl 1):S-719.

12. Kamada T, Murao T, Osawa M, et al. A total of 379 multiple white and flat elevated lesions in the gastric fundus are induced by acid suppressive agents and long-term proton pump inhibitor treatment. Gastroenterology 2015;148(4 Suppl 1):S-319.

13. Park YJ, Kim GH. Proton pump inhibitor-induced fundic gland polyposis. Korean J Intern Med 2017;32:197-198.

14. Hongo M, Fujimoto K. Incidence and risk factor of fundic gland polyp and hyperplastic polyp in long-term proton pump inhibitor therapy: a prospective study in Japan. J Gastroenterol 2010;45:618-624. 